\title{
DETERMINAÇÃO DE METAIS PESADOS EM SEDIMENTOS URBANOS POR FLUORESCÊNCIA DE RAIOS-X
}

\section{DETERMINATION OF HEAVY METALS IN URBAN SEDIMENTS BY X-RAY FLUORESCENCE}

\section{Emanoelle Pereira da Silva ${ }^{1}$, Eduardo Queija de Siqueira ${ }^{1}$, Fernando Schimidt ${ }^{2}$, Claudia de Souza Guedes ${ }^{1}$}

\author{
${ }^{1}$ Universidade Federal de Goiás, Escola de Engenharia Civil E-mail \\ eduqs@yahoo.com \\ ${ }^{2}$ Instituto Federal de Educação, Ciência e Tecnologia de Góias E-mail \\ schimidt99@gmail.com
}

\begin{abstract}
RESUMO
Os sedimentos urbanos depositados nas superfícies impermeáveis são uma fonte de poluição dos corpos de água no meio urbano. No entanto, poucos estudos têm sido desenvolvidos para verificar o potencial poluidor desses sedimentos. Assim, este artigo visa quantificar a concentração de elementos químicos, sobretudo de metais pesados, verificar a sua relação com o fluxo de veículos e traçar o perfil granulométrico dos sedimentos. Para tal, foram coletados sedimentos em três ruas da cidade de Goiânia, pelo método de aspiração por via úmida. Os elementos foram determinados por fluorescência de raios-X por energia dispersiva (EDXRF) e a granulometria analisada por difração a laser. Os resultados mostram altas concentrações de elementos tóxicos como Ba $(1098 \mathrm{mg} / \mathrm{kg}), \mathrm{Cr}$ $(174 \mathrm{mg} / \mathrm{kg})$ e Zn $(280 \mathrm{mg} / \mathrm{kg})$. Em geral, a ordem decrescente de concentração dos elementos foi Si $>\mathrm{Al}>\mathrm{Fe}>\mathrm{Ca}>\mathrm{K}>\mathrm{Ti}>\mathrm{S}>\mathrm{Ba}>\mathrm{Zn}>\mathrm{Mn}>\mathrm{Cr}>\mathrm{Sr}>\mathrm{Cu}>\mathrm{Zr}>\mathrm{V}>\mathrm{Au}>\mathrm{Ge}$. Os elementos analisados apresentaram um coeficiente de correlação de Pearson positivo para o fluxo de veículos, fato não verificado para Fe e Al. Da granulometria observou-se que mais de $60 \%$ das frações são < que $200 \mathrm{~m}$.
\end{abstract}

Palavras-chave: Sedimentos urbanos. Poluição difusa. Metais pesados. Fluorescência de Raios-X.

\begin{abstract}
Urban sediments deposited on paved surfaces are a source of pollution to water bodies. However, a few studies have been developed to check the potential pollution of these sediments. So this article aims to quantify the concentration of chemistry elements, above all heavy metals, verify the relation of those with the traffic flow and trace the sediments grain size distribution. For this, sediments were collected in three streets in Goiânia City, by the wet vacuuming method. The elements were determined by the energy dispersive X-ray fluorescence (EDXRF) and the particle size analyzed by laser diffraction. The results indicate high concentrations of toxic elements, as $\mathrm{Ba}(1098 \mathrm{mg} / \mathrm{kg}), \mathrm{Cr}$ $(174 \mathrm{mg} / \mathrm{kg})$ e $\mathrm{Zn}(280 \mathrm{mg} / \mathrm{kg})$. In general, the decreasing order of concentration elements were found $\mathrm{Al}>\mathrm{Fe}>\mathrm{Ca}>\mathrm{K}>\mathrm{Ti}>\mathrm{S}>\mathrm{Ba}>\mathrm{Zn}>\mathrm{Mn}>\mathrm{Cr}>\mathrm{Sr}>\mathrm{Cu}>\mathrm{Zr}>\mathrm{V}$. The elements analyzed
\end{abstract}


showed a positive Pearson coefficient correlation to the traffic flow, fact not verified to $\mathrm{Fe}$ and $\mathrm{Al}$. Particle sizes observed were more than $60 \%<$ than $200 \mathrm{~m}$.

Key-words: Urban sediments. Diffuse pollution. Heavy metals. X-Ray Fluorescence.

\section{INTRODUÇÃO}

Os sedimentos urbanos depositados nas superfícies impermeáveis, tais como nos telhados, ruas, avenidas e nos sistemas de drenagem são uma potencial fonte de deterioração da qualidade da água em bacias urbanas. Para Pitt e Burton (2001), as águas pluviais que lavam essas superfícies transportam uma carga de poluentes que pode ser maior que em alguns efluentes, podendo assim causar impactos ainda não mensurados aos corpos de água.

De acordo com Tucci (2003), os poluentes provêm principalmente da deposição atmosférica, da erosão do solo provocada pela velocidade do escoamento, desgaste e lavagem das superfícies, acúmulo, deposição e transporte de resíduos sólidos urbanos, atividades industriais e do tráfego de veículos. Destas principais fontes de poluição, Egodawatta (2007) comentou que o fluxo intenso de veículos nas áreas urbanas é um fator crítico que afeta a concentração dos poluentes depositados na superfície asfáltica, de forma que a maior proporção de elementos tóxicos depositados na superfície está relacionada com o tráfego de veículos.

Dos poluentes gerados pelas atividades urbanas estão, às partículas sólidas, patógenos, nutrientes, carbono orgânico, lixo e detritos, hidrocarbonetos e metais pesados. Dentre estes, os metais pesados são de grande relevância no estudo ambiental devido a sua toxicidade em ecossistemas aquáticos. Os metais comumente encontrados são o $\mathrm{Pb}, \mathrm{Zn}, \mathrm{Fe}, \mathrm{Cu}, \mathrm{Cd}, \mathrm{Cr}$ e $\mathrm{Ni}$, os quais são advindos principalmente da gasolina, desgaste de pneus e de revestimento de freios entre outros (EPA, 1999; PITT e BURTON, 2001; MIGUNTANNA, 2009).

Nesta dinâmica de transporte de sedimentos, vários estudos têm sido desenvolvidos os quais visam determinar a composição dos sedimentos e como são transportados. De acordo com Herngren (2005) e Gunawardana et al. (2011), a composição dos sedimentos varia com uma gama de fatores, tais como, localização geográfica (característica do solo), uso do solo e condições do tráfego. Esses fatores influenciam também na distribuição granulométrica das partículas dos sedimentos no ambiente urbano. Neste tipo de estudo, as partículas mais finas são as mais importantes para contaminação dos sedimentos (VIKLANDER, 1998), uma vez que são concentradoras de metais, devido à alta porosidade e capacidade de troca iônica da estrutura.

Uma das pesquisas pioneiras nesta área foi desenvolvida por Sartor e Boyd (1972) em 12 cidades dos Estados Unidos, que usando o método de aspiração a seco coletaram amostras em ruas com diferentes usos do solo e determinaram a composição e as cargas de poluentes coletadas nas ruas. Eles concluíram que $51,2 \%$ de todos os metais pesados e outros poluentes estão concentrados nas partículas $<246 \mathrm{~m}$, onde os metais encontrados em maiores concentrações foram $\mathrm{Zn} \mathrm{e} \mathrm{Pb}$.

Ball et al. (1998) em Sydney, Austrália usando o método de aspiração e escovação encontraram que a maior carga de poluentes está adsorvida nas partículas $<70$ m e os metais com maiores concentrações foram o $\mathrm{Fe}, \mathrm{Pb}$ e $\mathrm{Zn}$.

Deletic e Orr (2005) em Aberdeen, Escócia usando o método de aspiração úmida, analisaram a concentração de metais pesados e outros nutrientes. As maiores concentrações de metais pesados foram encontradas nas frações $<63 \mathrm{~m}$ para $\mathrm{Zn}, \mathrm{Pb}$ e $\mathrm{Cu}$.

Zhao et al. (2011) em Beijing, China usando aspiração a seco observaram que os metais pesados encontram-se, principalmente, depositados nas partículas $<250 \mathrm{~m}(80 \%)$, e que as partículas de maior importância no escoamento superficial são $<100 \mathrm{~m}$.

Ressalta-se que as concentrações e cargas dos poluentes em diversos estudos podem ter uma considerável variação, devido aos diferentes métodos de amostragem e métodos de análise 
(DELETIC e ORR, 2005). No entanto, estudos apontam que os sedimentos urbanos possuem altas concentrações de poluentes agregados as suas partículas, sobretudo de metais pesados, os quais são lixiviados das superfícies pela chuva e incorporados ao escoamento superficial, provocando assim poluição das águas pluviais e dos cursos de água.

Desta forma, o conhecimento dos poluentes, da quantidade e do tamanho das partículas é de suma importância para propor medidas compensatórias de conservação dos ecossistemas aquáticos e de subsistência humana, tais como subsídios para projetos de estruturas de tratamento, como bacias de sedimentação e detenção que atuem na remoção de poluentes das águas de drenagem. Neste contexto, este trabalho tem por objetivo quantificar elementos químicos, sobretudo metais pesados, presentes em sedimentos urbanos, avaliar a relação destes com o fluxo de veículos, verificar a massa acumulada durante os dias e traçar um perfil granulométrico dos sedimentos depositados em três superfícies asfálticas com diferentes tráfegos de veículos e uso do solo, da cidade de Goiânia-Goiás.

\section{MATERIAL E MÉTODOS}

\section{1. Área de Estudo}

O município de Goiânia com extensão de $733 \mathrm{~km}^{2}$ é a cidade mais populosa do Estado de Goiás, e com uma população estimada em 1.302.001 milhões de habitantes (IBGE, 2010). A capital tem como principal bioma o Cerrado, o relevo é basicamente constituído por planaltos em uma cota de $740 \mathrm{~m}$ com poucos declives onde o solo é arenoso e ácido. O clima é constituído por duas estações distintas com um período chuvoso e outro seco, onde a temperatura varia de $18{ }^{\circ} \mathrm{C}$ a $32{ }^{\circ} \mathrm{C}$ e a média de precipitação anual entre $1400 \mathrm{~mm}$ e $1500 \mathrm{~mm}$.

Em Goiânia foram selecionadas três ruas com distintos tráfegos de veículos e uso do solo. Para eleger locais propícios ao desenvolvimento da pesquisa alguns critérios foram considerados: o mínimo de perturbação para o tráfego de veículos da região e boa acessibilidade a pontos de água e energia. O fluxo de veículo foi determinado pela Ficha de Contagem Volumétrica I, do Manual de Estudos de Tráfego (BRASIL, 2006). A declividade foi determinada pelo programa X-Clinometer em três pontos para cada rua, e através destes foi calculado o valor médio. Para levantamento de dados do uso do solo, foi realizada uma pesquisa in loco com fotos. As ruas adotadas foram Avenida Universitária e Rua 240, ambas no Setor Universitário, e a Viela 1 no Parque Industrial.

\subsection{Amostragem do sedimento}

O material acumulado na superfície pavimentada foi coletado em campanhas entre agosto e setembro de 2012, usando a técnica de aspiração por via úmida, que consiste na lavagem da superfície com spray ou jato de água e simultânea aspiração. A técnica foi empregada por Bris et al. (1999) e Deletic e Orr (2005), os quais verificaram que a pressão da água contra a superfície desagrega as partículas finas da massa asfáltica, tornando-as mais viáveis de serem aspiradas. Desta forma, a técnica é mais eficiente na remoção de finos comparada a outros métodos como varrição, escovação e aspiração a seco.

As amostras foram coletadas em seis parcelas distribuídas ao longo de $200 \mathrm{~m}$ de cada rua durante 14 dias, sendo coletadas amostras no $1^{\circ}, 2^{\circ}, 3^{\circ}, 5^{\circ}, 7^{\circ}$ e $14^{\circ}$ dia antecedente a um evento de precipitação (Figura 1), em uma área de $0,5 \mathrm{~m}^{2}$ delimitada por uma moldura de madeira colocada entre a sarjeta e a faixa central da estrada. Os sedimentos foram coletados na faixa central, pois é onde se encontram as frações mais finas dos sedimentos e onde as substâncias que derramam ou vazam dos veículos sobre a superfície são geralmente encontrados em maiores concentrações (SARTOR e BOYD, 1972; GROTTKER, 1987). 


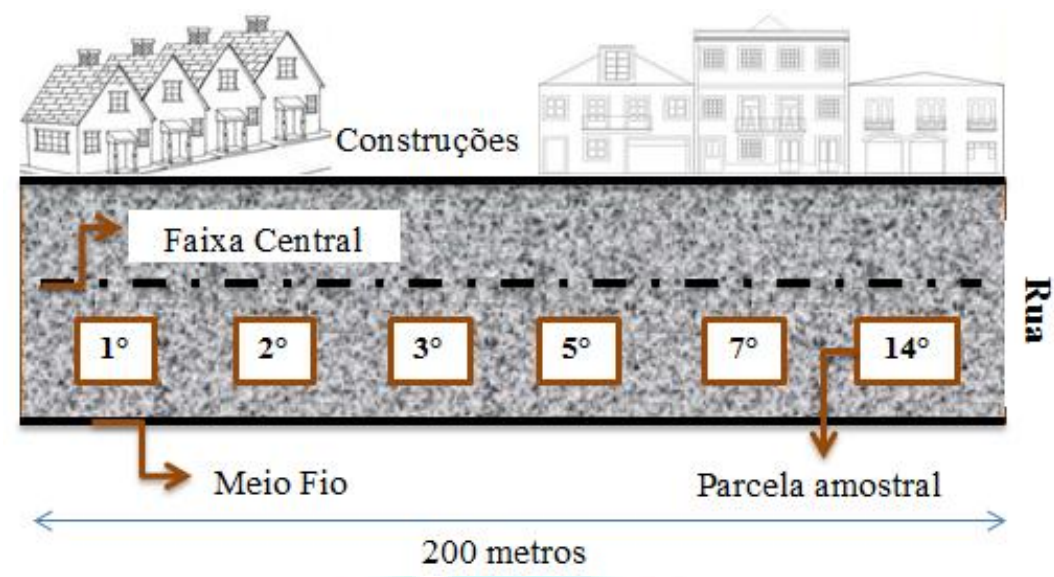

Figura 1. Mapa experimental da coleta do sedimento na rua.

Desta forma, sob cada superfície delimitada foi lançado 0,5 litros de água destilada com auxílio de um pulverizador, a qual foi simultaneamente aspirada por meio de um aspirador comercial, modelo Flex S com potência de $1400 \mathrm{~W}$, como ilustrado na Figura 2. As amostras coletada no interior do aspirador foram homogeneizadas e transferidas para frascos de coleta e encaminhadas para análise.

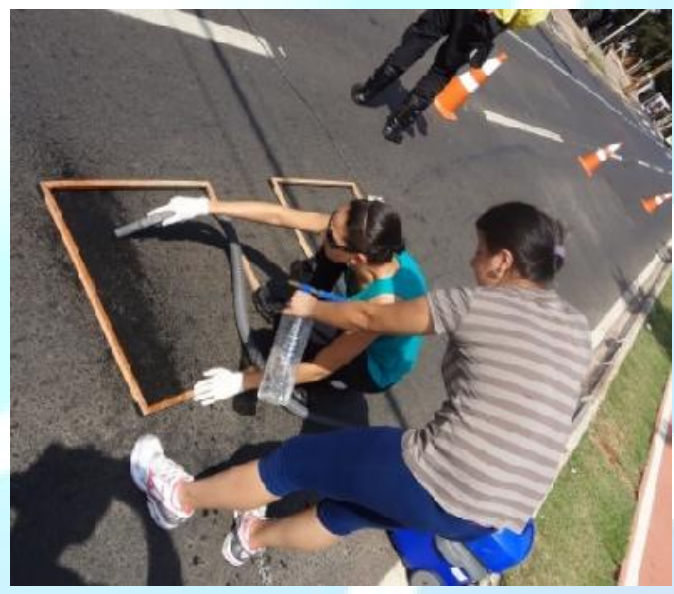

Figura 2. Aspectos gerais da coleta de sedimento na parcela amostral de $0,5 \mathrm{~m}^{2}$.

\subsection{Análise das amostras}

As amostras coletadas foram evaporadas em Banho-Maria e depois secas a $105{ }^{\circ} \mathrm{C} \pm 3{ }^{\circ} \mathrm{C}$ por $24 \mathrm{~h}$ em estufa de secagem para remover a umidade, e a determinação da massa foi por gravimetria em balança analítica de precisão. Em seguida, os sedimentos foram agrupados para maior representatividade amostral de cada rua e analisados quanto à composição química por Fluorescência de Raios-X por Energia Dispersiva, do inglês EDXRF.

A técnica EDXRF se baseia na medida da intensidade dos raios-X característicos dos elementos químicos, emitidos pelos componentes da amostra. De acordo com Yeung et al. (2003), é um método rápido, simples e não destrutivo para a análise de diversos elementos traços, sendo uma das técnicas mais usadas na geoquímica na análise de solo e sedimentos.

Das amostras de cada rua foram retiradas cerca de $3 \mathrm{~g}$ para análise por EDXRF. As análises foram feitas em duplicata e foram analisados 17 elementos ( $\mathrm{Si}, \mathrm{Al}, \mathrm{Fe}, \mathrm{Ca}, \mathrm{K}, \mathrm{Ti}, \mathrm{S}, \mathrm{Ba}, \mathrm{Zn}, \mathrm{Mn}, \mathrm{Cr}$, $\mathrm{Sr}, \mathrm{Cu}, \mathrm{Zr}, \mathrm{V}, \mathrm{Au}, \mathrm{Ge}$ ) utilizando o método de Parâmetros Fundamentais. Os espectros foram obtidos pelo Espectrofotômetro SHIMADZU, modelo EDX-720 em ambiente de vácuo, e as análises foram conduzidas no laboratório GERX do IQ-UNICAMP. 
A matéria orgânica foi determinada por calcinação a $440^{\circ} \mathrm{C}$ por 4 horas (EPA, 2002).

Para determinar a granulometria, as amostras foram previamente peneiradas em frações maiores que $1000 \mathrm{~m}$ e $500 \mathrm{~m}$ em peneiras de material não metálico. O material retido de cada peneira foi pesado e armazenado separadamente. Da massa passante da peneira de $500 \mathrm{~m}$ foi retirada cerca de $2 \mathrm{~g}$, a qual foi avaliada em um analisador de partículas por difração a laser da Malvern, modelo HYDRO 2000MU pelo software Mastersize 2000, no Laboratório de Geomorfologia, Pedologia e Geografia Física (LABOGEF) do Instituto de Ciências SócioAmbientais da UFG. O procedimento analítico consiste em adicionar um percentual da amostra em um Becker preenchido com cerca de $300 \mathrm{~mL}$ de água destilada. Neste ambiente o módulo ultrassom e velocidade de rotação dispersa as partículas e quebra os flocos. Os dados obtidos de distribuição foram tratados no GRADISTAT, um programa estatístico de análise de sedimentos (BLOTT e PYE, 2001).

\section{RESULTADOS E DISCUSSÃO}

\subsection{Características dos sedimentos}

$\mathrm{Na}$ Tabela 1, são apresentadas as características de cada rua amostrada, a carga de sedimentos acumulada e quantidade de matéria orgânica.

Verifica-se que as ruas possuem características diferenciadas em relação ao uso do solo e tráfego de veículos. A Avenida Universitária apresenta maior tráfego, seguida da Rua 240 e da Viela 1. O serviço de varrição também é uma característica importante, pois interfere na distribuição das partículas e no acúmulo dos poluentes nas superfícies. Esse atua removendo as partículas grosseiras e dispersando as mais finas, tornando-as mais suscetíveis de serem transportadas pelo vento e chuva (VAZE e CHIEW, 2002).

Da carga de sedimentos, observou-se uma relação inversa com o fluxo de veículos, sendo coletada uma carga de $85,8 \mathrm{~g} / \mathrm{m}^{2}$ para a Avenida Universitária, 246, $4 \mathrm{~g} / \mathrm{m}^{2}$ para Rua 240 e 361,5 $\mathrm{g} / \mathrm{m}^{2}$ para Viela 1 . No entanto, deve-se observar que a rua que obtém maior carga de sedimentos não possui o serviço de limpeza, o que colabora com o maior aporte de sedimentos.

$\mathrm{Na}$ Avenida Universitária a variação da carga de sedimentos foi de 8,8 a 21,6 g/m² com uma média de 14,3 g/m², na Rua 240 esta faixa foi de 27,7 a $67,9 \mathrm{~g} / \mathrm{m}^{2} \mathrm{com}$ uma média de $41,1 \mathrm{~g} / \mathrm{m}^{2}$ e para Viela 1 a variação foi de 11,6 a $80,1 \mathrm{~g} / \mathrm{m}^{2}$ com média de $46,9 \mathrm{~g} / \mathrm{m}^{2}$.

Tabela 1 - Descrição da área de estudo e características do sedimento das ruas estudadas

\begin{tabular}{c|c|c|c}
\hline Local & Av. Universitária & Rua 240 & Viela 1 \\
\hline $\begin{array}{c}\text { VMD* } \\
\text { (veículos/dia) }\end{array}$ & 10000 & 1500 & 150 \\
\hline $\begin{array}{c}\text { Declividade } \\
\text { média }(\%)\end{array}$ & 2,5 & 3,7 & 2,7 \\
\hline Uso do Solo & $\begin{array}{l}\text { Comercial; } \\
\text { Circulação de ônibus } \\
\text { e caminhões; } \\
\text { Plantas ornamentais; } \\
\text { Pouco solo exposto; }\end{array}$ & $\begin{array}{l}\text { Residencial e comercial; } \\
\text { Circulação de caminhões; } \\
\text { Muitas árvores; } \\
\text { Muito solo exposto; }\end{array}$ & $\begin{array}{l}\text { Residencial; } \\
\text { Pouca circulação de } \\
\text { caminhões; } \\
\text { Poucas árvores; } \\
\text { Pouco solo exposto; }\end{array}$ \\
\hline $\begin{array}{c}\text { Serviço de } \\
\text { varrição }\end{array}$ & $\begin{array}{c}\text { Duas vezes por } \\
\text { semana }\end{array}$ & Uma vez por semana & Esporadicamente \\
\hline $\begin{array}{c}\text { Carga de } \\
\text { sedimento }\left(\mathrm{g} / \mathrm{m}^{2}\right)\end{array}$ & 85,8 & 246,4 & 361,5 \\
\hline $\begin{array}{c}\text { Matéria } \\
\text { orgânica }(\%)\end{array}$ & 7,2 & 20,9 & 6,1 \\
\hline
\end{tabular}

${ }^{*}$ Volume médio diário; * Carga total coletada nas 6 parcelas; 
Resultados similares foram encontrados por Vaze e Chiew (2002), em uma rua próxima ao maior centro de negócios de Melbourne, Austrália, onde a carga de sedimentos que variou de 5,0 a $55,0 \mathrm{~g} / \mathrm{m}^{2}$. Já nos estudos de Deletic e Orr (2005) Aberdeen, Escócia, em uma rua de uso residencial com circulação de diversos veículos e ônibus, a carga média de sedimentos foi de $101,9 \mathrm{~g} / \mathrm{m}^{2}$, mais que o dobro da detectada neste estudo. Dotto (2006) em Santa Maria, Brasil encontrou para ruas residenciais uma variação de 4 a $350 \mathrm{~g} / \mathrm{m}^{2}$ na Rua Marquês do Herval e de 10 a $240 \mathrm{~g} / \mathrm{m}^{2}$ na Rua Rigobeto Duarte.

Em relação à matéria orgânica, observa-se que a Rua 240 possui a maior concentração (20,9\%), fato que pode estar correlacionado com a maior densidade de árvores presentes na rua em relação às outras ruas amostradas.

\subsection{Distribuição Granulométrica}

Nas Figuras 3 e 4 estão apresentadas as frações de sedimentos de cada rua e a curva de distribuição. Na Figura 3 observa-se que para Avenida Universitária e Rua 240 mais de $50 \%$ dos sedimentos são < $500 \mathrm{~m}$; na Viela 1 esse montante é de 33,7\% e a fração com maior volume foi a $>1000 \mathrm{~m}$ com 51,7\%. Desta forma, os sedimentos mais grosseiros, ou seja, maiores que $1000 \mathrm{~m}$ foram encontrados em maior concentração na sequência Viela 1, Rua 240 e Avenida Universitária respectivamente. Fato que aponta, quanto menor o fluxo de veículos maior as frações dos sedimentos encontradas nas vias, isto também aliado ao fator da varrição.

A Figura 4 expressa a distribuição granulométrica das frações menores que $500 \mathrm{~m}$, na qual se pode observar que esses sedimentos são compostos predominantemente de argila, silte e areia fina, ou seja, a distribuição das partículas está entre 1-250 m. Sendo que, pelos dados obtidos no GRADISTAT, 67,1\%, 66,9\% e 78,0\% das frações dos sedimentos são < $250 \mathrm{~m}$ respectivamente para Avenida Universitária, Rua 240 e Viela 1. E ainda destas frações, 25,4\%, 28,4\% e 41,5 são < $63 \mathrm{~m}$, ou seja, estão entre argila e silte. A distribuição da Avenida Universitária é unimodal, com pico em $300 \mathrm{~m}$ e da Rua 240 e Viela 1 trimodal, com picos nas frações de silte e areia média. Os diâmetros médios $\mathrm{D}_{50}$ são $153,7 \mathrm{~m}, 137,1 \mathrm{~m}$ e 85,2 m, respectivamente, para Avenida Universitária, Rua 240 e Viela 1.

Diversos estudos (WANG et al., 2006; HERNGREN et al., 2006; SUTHERLAND et al., 2012) apontam que as partículas finas têm importante papel na contaminação dos sedimentos urbanos, pois são concentradoras de metais pesados e tem a capacidade de estarem em suspensão e de serem transportadas a longas distâncias pelo escoamento superficial, representando assim riscos aos recursos hídricos.

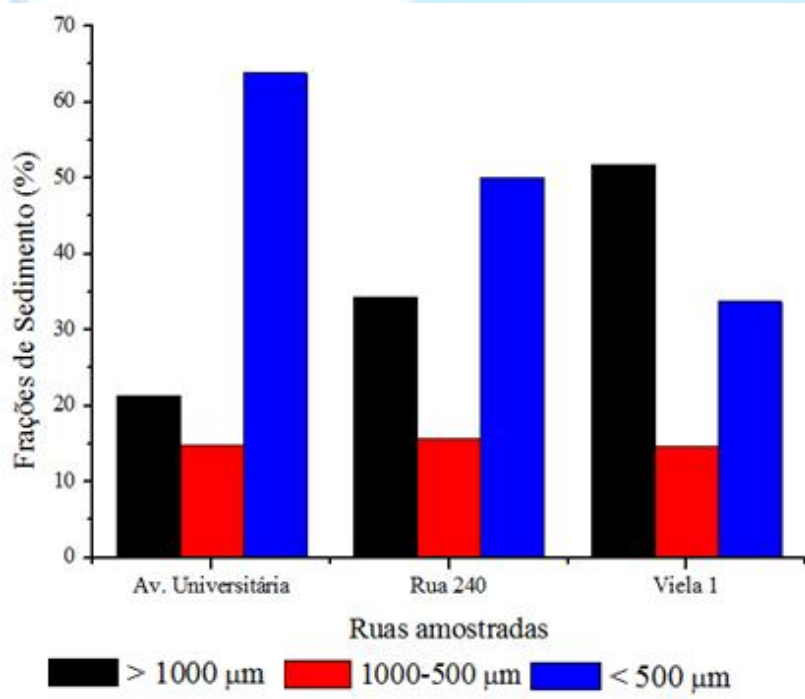

Figura 3. Frações dos sedimentos nas ruas Avenida Universitária, 240 e Viela 1.

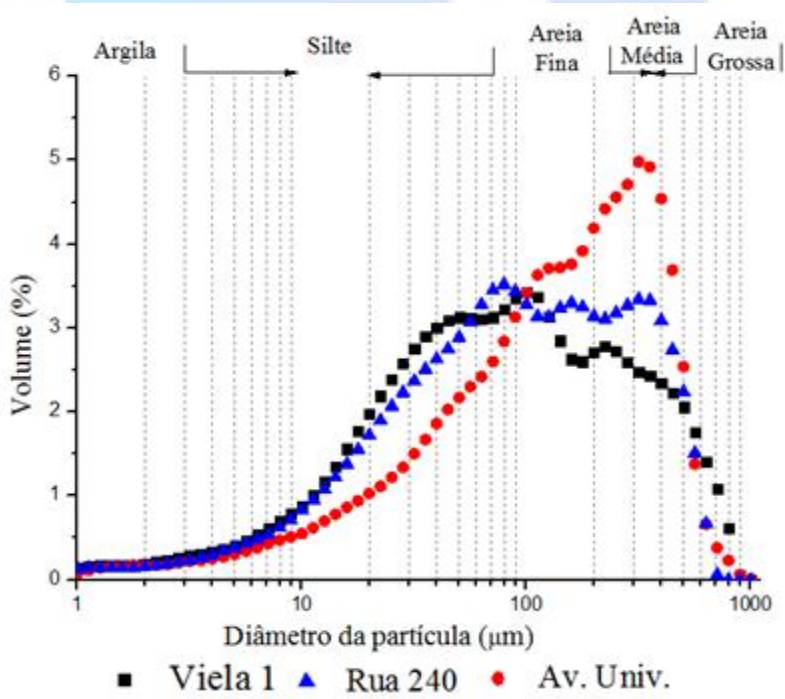

Figura 4. Distribuição Granulométrica dos sedimentos nas ruas estudadas. 


\subsection{Concentração de metais}

As concentrações dos 17 elementos detectados nas amostras de sedimento são apresentadas na Tabela 2. Nesta se pode observar que todas as ruas apresentam concentrações de metais, dos quais muitos são considerados tóxicos ao meio ambiente (EPA, 1999; VOLESKY, 2001). Também é possível observar uma relação dos metais com o fluxo de veículos (Tabela 3), pois as maiores concentrações dos elementos analisados foram encontradas na Avenida Universitária, sobretudo para os metais $\mathrm{Ca}, \mathrm{K}, \mathrm{Ti}, \mathrm{Ba}, \mathrm{Zn}, \mathrm{Mn}, \mathrm{Cr}$ e $\mathrm{Cu}$, dos quais devem-se destacar as concentrações de $1098 \mathrm{mg} / \mathrm{kg}, 280 \mathrm{mg} / \mathrm{kg}, 174 \mathrm{mg} / \mathrm{kg}$ e $92 \mathrm{mg} / \mathrm{kg}$ respectivamente para $\mathrm{Ba}, \mathrm{Zn}, \mathrm{Cr}$ e Cu, os quais apresentam grande relevância na poluição ambiental. Sendo ainda, importante ressaltar que elementos como $\mathrm{Ba}$ e $\mathrm{Cr}$ só foram detectados na rua com maior fluxo de veículos.

Tabela 2 - Concentração média dos elementos químicos detectados nos sedimentos das ruas estudadas, em $\mathrm{mg} / \mathrm{kg}$, e comparação com outros estudos

\begin{tabular}{c|c|c|c|c|c|c}
\hline Elementos & $\begin{array}{l}\text { Av. Univ. } \\
\text { Neste } \\
\text { estudo }\end{array}$ & $\begin{array}{l}\text { Rua 240 } \\
\text { Neste } \\
\text { estudo }\end{array}$ & $\begin{array}{c}\text { Viela } 1 \\
\text { Neste } \\
\text { estudo }\end{array}$ & $\begin{array}{c}\text { Mong Kok } \\
\text { Yeung et al., } \\
(2003)\end{array}$ & $\begin{array}{c}\text { Tai Po } \\
\text { Yeung et al., } \\
(2003)\end{array}$ & $\begin{array}{c}\text { Xianyang } \\
\text { Shi et al., } \\
(2013)\end{array}$ \\
\hline $\mathrm{Si}$ & 50107 & 29882 & 42347 & 158000 & 211000 & - \\
$\mathrm{Al}$ & $\mathbf{3 6 1 1 3}$ & 22565 & 33304 & 13900 & 24100 & - \\
$\mathrm{Fe}$ & 18661 & 10672 & 23020 & 14100 & 12.500 & - \\
$\mathrm{Ca}$ & 15480 & 13729 & 13903 & 287000 & 253000 & - \\
$\mathrm{K}$ & 6832 & 5224 & 6165 & 14100 & 10600 & - \\
$\mathrm{Ti}$ & 2466 & 1458 & 2330 & 2970 & 2340 & - \\
$\mathrm{S}$ & 1761 & 1382 & 1243 & - & - & - \\
$\mathrm{Ba}$ & $\mathbf{1 0 9 8}$ & nd & nd & 299 & 126 & - \\
$\mathrm{Zn}$ & $\mathbf{2 8 0}$ & 111 & 203 & 4860 & 3510 & 375 \\
$\mathrm{Mn}$ & 226 & 146 & 194 & 512 & 664 & 604 \\
$\mathrm{Cr}$ & $\mathbf{1 7 4}$ & nd & nd & 177 & 65 & 136 \\
$\mathrm{Sr}$ & 105 & 52 & 110 & 144 & 153 & - \\
$\mathrm{Cu}$ & $\mathbf{9 2}$ & 54 & 72 & 133 & 40 & 132 \\
$\mathrm{Zr}$ & 66 & 23 & 97 & 180 & 250 & - \\
$\mathrm{V}$ & 47 & 67 & 85 & 46 & 37 & - \\
$\mathrm{Au}$ & nd & nd & 48 & - & - & - \\
$\mathrm{Ge}$ & nd & nd & 42 & - & - & - \\
\hline
\end{tabular}

*Não detectado

$\mathrm{Na}$ Viela 1 foram detectadas as maiores concentrações para $\mathrm{Fe}, \mathrm{Sr}, \mathrm{Zr}$ e $\mathrm{V}$, sendo ainda encontrados os elementos $\mathrm{Au}$ e $\mathrm{Ge}$, os quais não foram observados nas outras ruas estudas. A Rua 240 apresentou concentrações intermediárias entre Avenida Universitária e Viela 1.

Yeung et al. (2003), encontraram concentrações similares para Al, $\mathrm{Fe}, \mathrm{Ti}, \mathrm{Cr}, \mathrm{Cu}, \mathrm{Sr}$ e $\mathrm{V}$ em duas ruas de uso comercial e residencial em Hong Kong. Estes dados também são apresentados na Tabela 2 para comparação. Para $\mathrm{Cr}$ e $\mathrm{Cu}$ os resultados da Rua Mong Kok se assemelham aos da Avenida Universitária. Shi et al. (2013), em ruas da cidade de Xianyang encontraram concentrações superiores para $\mathrm{Cu}, \mathrm{Zn}, \mathrm{Cr}$ e $\mathrm{Mn}$.

$\mathrm{Na}$ Tabela 3 são apresentados os coeficientes de correlação de Pearson $\left(\mathrm{r}^{2}\right)$ dos metais com o fluxo de veículos (FV). Os coeficientes indicam que os metais analisados possuem uma correlação positiva com o fluxo de veículos, sendo que os metais com melhor correlação foram $\mathrm{Ba}=\mathrm{Cr}>\mathrm{Cu}>$ $\mathrm{Zn}>\mathrm{Mn}$. O Fe e Al não apresentaram uma boa correlação. 
Tabela 3 - Coeficiente de correlação dos metais com o fluxo de veículos das ruas, Avenida Universitária, Rua 240 e Viela 1

\begin{tabular}{lllllllll}
\hline & $\mathbf{F V}$ & $\mathbf{A l}$ & $\mathbf{F e}$ & $\mathbf{B a}$ & $\mathbf{Z n}$ & $\mathbf{M n}$ & $\mathbf{C r}$ & $\mathbf{C u}$ \\
\hline $\mathbf{F V}$ & 1,00 & & & & & & & \\
$\mathbf{A l}$ & 0,56 & 1,00 & & & & & & \\
$\mathbf{F e}$ & 0,04 & $0,85^{*}$ & 1,00 & & & & & \\
$\mathbf{B a}$ & $0,99^{*}$ & $0,66^{* *}$ & 0,17 & 1,00 & & & & \\
$\mathbf{Z n}$ & $0,76^{* *}$ & $0,96^{*}$ & 0,68 & 0,84 & 1,00 & & & \\
$\mathbf{M n}$ & $0,72^{* *}$ & $0,98^{*}$ & 0,72 & 0,80 & 1,00 & 1,00 & & \\
$\mathbf{C r}$ & $0,99^{*}$ & $0,66^{* *}$ & 0,17 & 1,00 & 0,84 & 0,80 & 1,00 & \\
$\mathbf{C u}$ & $0,82^{*}$ & $0,93^{*}$ & 0,61 & 0,88 & 1,00 & 0,99 & 0,88 & 1,00 \\
\hline
\end{tabular}

$0,8 \geq \mathrm{r} \leq 1$ forte positiva; ${ }^{* *} 0,5 \geq \mathrm{r}<0,8$ moderada positiva

Alhassan et al. (2012), encontraram correlações positivas entre o fluxo de veículos e as concentrações de $\mathrm{Zn}, \mathrm{Cr}$, Fe e $\mathrm{Mn}$, no entanto o resultado não foi igual para todas as ruas, por exemplo, a rua Maiduguri obteve uma correlação insignificante para o $\mathrm{Fe}$, como observado neste trabalho. Isso possivelmente está relacionado com o solo das regiões amostradas possuírem altas concentrações de ferro. Alguns elementos também apresentaram correlações significativas entre si, como o $\mathrm{Cu}$ e $\mathrm{Mn}$ e $\mathrm{Cu}$ e $\mathrm{Zn}$.

\section{CONCLUSÕES}

- Os resultados apresentados indicam que os sedimentos urbanos encontrados nas ruas da cidade de Goiânia, contêm altas concentrações de elementos metálicos, sobretudo de metais pesados de relevância na poluição ambiental, como Fe, Ti, $\mathrm{Ba}, \mathrm{Zn}, \mathrm{Mn}, \mathrm{Cr}$ e $\mathrm{Cu}$.

- Observou-se que a maioria dos elementos químicos apresenta uma relação direta com o fluxo de veículos, sendo que, as maiores concentrações de elementos como $\mathrm{Ba}, \mathrm{Zn}, \mathrm{Cr}$ e $\mathrm{Cu}$, foram detectadas na rua com maior fluxo de veículos, sugerindo assim a natureza antropogênica destes poluentes. Esta relação não foi observada para os elementos $\mathrm{Fe}, \mathrm{Si}, \mathrm{Ca}$ e K, possivelmente, porque estes estão relacionados com a composição do solo local.

- Da carga de sedimentos acumulada durante os dias, observou-se esta tende a decrescer com o aumento do fluxo de veículos, na ordem Av. Universitária < Rua 240 < Viela 1, e que também depende de características físicas da área de estudo, como uso do solo, serviço de limpeza e densidade de árvores.

- Do perfil granulométrico dos sedimentos, observou-se que em geral mais de $50 \%$ das frações de sedimento são $<500 \mathrm{~m}$, e destas mais $60 \%$ são $<200 \mathrm{~m}$, ou seja, estão entre areia fina e argila, partículas de menor diâmetro e de grande relevância por serem complexadoras de metais e outros poluentes.

\section{AGRADECIMENTOS}

Agradecemos ao CNPq pela bolsa concedida que possibilitou o desenvolvimento da pesquisa. Aos laboratórios GERX-IQ da Unicamp, na pessoa da Professora Dra. Maria Isabel Bueno, e ao LABOGEF-IESA da UFG, na pessoa do professor Dr. Maximiliano Bayer. 


\section{REFERÊNCIAS}

ALHASSAN, A.J.; SULE, M.S.; ATIKU, M.K.; WUDIL, A.M.; DANGAMBO, M.A.; MASHI, J.A.; IBRAHIM, N.A. Study of Correlation Between Heavy Metal Concentration, Street Dust and Level of Traffic in Major Roads of Kano Metropolis, Nigeria. Nigerian Journal of Basic and Applied Science, v. 20, p. 161-168, 2012.

BALL, J.E.; JENKS, R.; AUBOURG, D. An assessment of the availability of pollutant constituents on road surfaces. The Science of the Total Environment, v. 209, p. 243-254, 1998.

BLOTT, S.J.; PYE, K. GRADISTAT: A Grain Size Distribution And Statistics Package For The Analysis Of Unconsolidated Sediments. Earth Surface Processes and Landforms, v. 26, p. 12371248, 2001.

BRASIL. Departamento Nacional de Infraestrutura de Transportes - DNIT. Manual de estudos de tráfego. Rio de Janeiro, 2006. 384 p. Disponível em:<

http://ipr.dnit.gov.br/manuais/manual_estudos_trafego.pdf>. Acesso em: nov. 2013.

BRIS, F.J.; GARNAUD, S.; APPERRY, N.; GONZALEZ, A.; MOUCHEL, J.M.; CHEBBO, G.;THÉVENOT, D.R A street deposit sampling method for metal and hydrocarbon contamination assessment. The Science of the Total Environment, v. 235, p. 211-220, 1999.

DELETIC, A.; ORR, D.W. Pollution Buildup on Road Surfaces. Journal of the Environmental Engineering, v.131, p.149- 159, 2005.

DOTTO, C.B.S. Acumulação e balanço de sedimentos em superfícies asfálticas em área urbana de Santa Maria- RS. 2006. 126p. Dissertação de Mestrado de Engenharia CivilUniversidade Federal de Santa Maria, Santa Maria-RS, 2006.

EGODAWATTA, P. Translation of small-plot scale pollutant build-up and wash-off measurements to urban catchment scale. Thesis submitted to Faculty of Built Environment and Engineering, Australia, 2007.

EPA. Preliminary Data Summary of Urban Storm Water Best Management Practices. EPA821-R-99-012. Office of Water (4303). Washington, 1999.

EPA. Methods for the determination of total organic carbon (TOC) in soils and sediments. Washington, 2002.

GROTTKER, M. Runoff quality from a street with medium traffic Loading. Science of the Total Environment, v. 59, p. 457- 466, 1987.

GUNAWARDANA, C.; GOONETILLEKE, A.; EGODAWATTA, P.; DAWES, L. Composition and source identification of road deposited pollutants. Proceedings of the First International Postgraduate Conference on Engineering, Designing and Developing the Built Environment for Sustainable Wellbeing, Faculty of Built Environment and Engineering, Queensland University of Technology, Queensland University of Technology, Brisbane, Australia, p. 151-156, 2011. 
HERNGREN, L. Build-up and wash-off process kinetics of PAHs and heavy metals on paved surfaces using a simulated rainfall. Thesis submitted to Faculty of Built Environment and Engineering, Australia, 2005.

HERNGREN, L; GOONETILLEKE, A.; AYOKO, G.A. Analysis of heavy metals in roaddeposited sediments. Analytica Chimica Acta, v. 571, p. 270-278, 2006.

IBGE- Instituto Brasileiro de Geografia e Estatística. Censo demográfico 2010. 2011. Disponível em< http://www.ibge.gov.br/home/presidencia/noticias/imprensa/ppts/0000000402.pdf>. Acesso em: 30 out. 2012.

MIGUNTANNA, N.P. Nutrients build-up and wash-off process in urban land uses. Thesis submitted to Faculty of Built Environment and Engineering, Australia, 2009.

PITT, A.; BURTON, R. Stormwater effects handbook: a toolbox for watershed managers, scientists, and engineers. Ed. CRC Press, New York, 2001.

SARTOR, J.D.; BOYD, G.B. Water pollution aspects of street surface contaminants. Office of Research and Monitoring U.S. Environmental Protection Agency - EPA, Washington, 1972.

SHI, X; CHEN, L; WANG, J. Multivariate analysis of heavy metal pollution in street dusts of Xianyang city, NW China. Environmental Earth Science, v. 69, p. 1973 -1979, 2013.

SUTHERLAND, R.A.; TACK, F.M.G.; ZIEGLER, A.D. Road-deposited sediments in an urban environment: A first look at sequentially extracted element loads in grain size fractions. Journal of Hazardous Materials, v. 225, p. 54-56, 2012.

TUCCI, C.E.M. Drenagem Urbana. Ciência e Cultura. São Paulo Oct. /Dec. v.55, n.4, 2003.

VAZE, J.; CHIEW, F.H.S. Experimental study of pollutant accumulation on an urban

Road surface. Urban Water, v. 4, p. 379-389, 2002.

VIKLANDER, M. Particle size distribution and metal content in street sediments. Journal of Environmental Engineering, v. 124, p. 761-766, 1998.

VOLESKY, B. Detoxification of metal-bearing effluents: biosorption for the next century. Hydrometallurgy, v. 59, p. 203-216, 2001.

WANG, X.S.; QIN, Y.; CHEN, Y.K. Heavy meals in urban roadside soils, part 1:effect of particle size fractions on heavy metals partitioning. Environmental Geology, v. 50, p.1061-1066, 2006.

YEUNG, Z.L.L.; KWOK, R.C.W. ;YU, K.N. Determination of multi-element profiles of street dust using Energy Dispersive X-Ray Fluorescence (EDXRF). Radiation and Isotopes, v. 58, p. 339346, 2003.

ZHAO, H.; LI, X.; WANG, X. Heavy Metal Contents of Road-Deposited Sediment along the Urban-Rural Gradient around Beijing and its Potential Contribution to Runoff Pollution.

Environmental Science and Technology, v. 45, p. 7120-7127, 2011. 